\title{
Periodic Tempered Distributions of Beurling Type and Periodic Ultradifferentiable Functions with Arbitrary Support
}

\author{
Byung Keun Sohn (iD \\ Department of Applied Mathematics, Inje University, Gimhae, Gyeongnam 50834, Republic of Korea \\ Correspondence should be addressed to Byung Keun Sohn; mathsohn@inje.ac.kr
}

Received 27 December 2017; Revised 13 March 2018; Accepted 31 March 2018; Published 9 May 2018

Academic Editor: Jean Michel Rakotoson

Copyright (C) 2018 Byung Keun Sohn. This is an open access article distributed under the Creative Commons Attribution License, which permits unrestricted use, distribution, and reproduction in any medium, provided the original work is properly cited.

Let $\delta_{\omega}^{\prime}(R)$ be the space of tempered distributions of Beurling type with test function space $\delta_{\omega}(R)$ and let $\mathscr{E}_{\omega, p}$ be the space of ultradifferentiable functions with arbitrary support having a period $p$. We show that $\mathscr{E}_{\omega, p}$ is generated by $\mathcal{S}_{\omega}(R)$. Also, we show that the mapping $\delta_{\omega}(R) \rightarrow \mathscr{E}_{\omega, p}$ is linear, onto, and continuous and the mapping $\mathcal{S}_{\omega, p}^{\prime}(R) \rightarrow \mathscr{E}_{\omega, p}^{\prime}$ is linear and onto where $\delta_{\omega, p}^{\prime}(R)$ is the subspace of $\mathcal{S}_{\omega}^{\prime}(R)$ having a period $p$ and $\mathscr{E}_{\omega, p}^{\prime}$ is the dual space of $\mathscr{E}_{\omega, p}$.

\section{Introduction}

In his excellent book [1], Zemanian introduced a space $\mathscr{P}_{T}$ of periodic test functions of period $T$ and a space $\mathscr{P}_{T}^{\prime}$ of periodic distributions with period $T$. He defined $T$ convolution, a different type of classical convolution, for $\mathscr{P}_{T}^{\prime}$ and discussed Fourier series of $\mathscr{P}_{T}^{\prime}$. His results have been applied in distribution theory [2-4], transform analysis [3,5], differential equations $[2,6,7]$, and signal analysis $[8,9]$.

Since then, developments of periodic ultradistributions and periodic ultradifferentiable functions theory have been made. Gorbačuk characterized two periodic ultradistributions $\mathscr{D}^{\prime}\left\{m_{k}\right\}$ and $\mathscr{D}^{\prime}\left(m_{k}\right)$ of Roumieu type and Beurling type, respectively, by the growth rate of their coefficients in [10] and Pilipović gave structure theorem for $\mathscr{D}^{\prime}\left\{m_{k}\right\}$ and $\mathscr{D}^{\prime}\left(m_{k}\right)$ in [11]. Also, Taguchi studied the Fourier coefficients of ultradifferentiable functions of Gevrey classes and ultradistributions in [12].

Gorbačuk, Pilipović, and Taguchi's results have been applied in almost periodic ultradistributions of Roumieu type and Beurling type $[13,14]$ and signal analysis [15].

In the meantime, Smith discussed tempered distributions by focusing on distributional convolution and some of its application to signal analysis in [16] and showed the relationship between periodic tempered distributions, $\mathcal{S}_{1}^{\prime}$, and dual space of the smooth periodic functions, $P_{p}^{*}$, in [17]. Smith also discussed the convolution for $\delta_{1}^{\prime}$ and $P_{p}^{*}$ and Fourier series of $\mathcal{S}_{1}^{\prime}$ in [17].

In this paper, we will consider periodic tempered distributions of Beurling type, $\mathcal{S}_{\omega, p}^{\prime}(R)$, which is an extension of periodic tempered distributions and periodic ultradifferentiable functions with arbitrary support, $\mathscr{E}_{\omega, p}$. We show that $\mathscr{E}_{\omega, p}$ is generated by $\mathcal{S}_{\omega}(R)$ which is a test function space of tempered distributions of Beurling type, $\delta_{\omega}^{\prime}(R)$, and the mapping $\mathcal{S}_{\omega}(R) \rightarrow \mathscr{E}_{\omega, p}$ is linear, onto, and continuous. Also we show that the mapping $\mathcal{S}_{\omega, p}^{\prime}(R) \rightarrow \mathscr{E}_{\omega, p}^{\prime}$ is linear and onto, where $\mathscr{E}_{\omega, p}^{\prime}$ is a dual space of $\mathscr{E}_{\omega, p}$.

Our results are indebted to Zemanian [1] and especially to Smith [17].

\section{Periodic Tempered Distributions of Beurling Type and Periodic Ultradifferentiable Functions}

We review tempered distributions of Beurling type and ultradifferentiable functions and related results which we need later. For more details, we can refer to [18-20].

We denote by $\mathfrak{M}_{c}$ the set of all continuous functions $\omega$ on $R$ satisfying the following conditions:

$(\alpha) \quad 0=\omega(0) \leq \omega(\xi+\eta) \leq \omega(\xi)+\omega(\eta) ;$ 
( $\beta) \int\left(\omega(\xi) /(1+|\xi|)^{2}\right) d \xi<\infty$;

$(\gamma) \omega(\xi) \geq a+b \log (1+|\xi|)$ for some real $a$ and positive $b$;

( $\delta) \omega(\xi)=\Omega(|\xi|)$, where $\Omega$ is the increasing continuous concave function such that $\max _{|\xi| \leq r} \omega(\xi) \leq \Omega(r)$.

Example 1. $\omega(\xi)=\log (1+|\xi|), \omega(\xi)=|\xi|^{1 / d}(d>1)$.

More detailed properties and examples of $\omega$ can be found in [21] and $\omega$ represents an element in $\mathfrak{M}_{c}$ throughout this paper.

Let $\phi \in L_{1}(R)$ and if $\lambda$ is a real number, we write

$$
\|\phi\|_{\lambda}^{(\omega)}=\int|\widehat{\phi}(\xi)| e^{\lambda \omega(\xi)} d \xi
$$

which may be finite or infinite.

Definition 2. Let $\mathscr{D}_{\omega}(R)$ be the set of all $\phi \in L_{1}(R)$ such that $\phi$ has compact support and $\|\phi\|_{\lambda}^{(\omega)}<\infty$ for all $\lambda>0$. The continuous linear functional of $\mathscr{D}_{\omega}(R)$ is denoted by $\mathscr{D}_{\omega}^{\prime}(R)$ whose elements are called the distribution of Beurling type.

For each $\lambda>0,\|\cdot\|_{\lambda}^{(\omega)}$ defines the seminorms in $\mathscr{D}_{\omega}(R)$. From [19, Proposition 1.3.6], if $\omega(\xi)=\log (1+|\xi|)$, then $\mathscr{D}_{\omega}(R)=C_{c}^{\infty}(R)$, where $C_{c}^{\infty}(R)$ is the set of all infinitely differentiable functions with compact support in $R$. Also from [19, Theorem 1.3.18] and the property $(\gamma)$ of $\omega$, we have $\mathscr{D}_{\omega}(R) \subset C_{c}^{\infty}(R)$. A sequence $\left\{\phi_{n}\right\}$ is said to converge to $\phi$ in $\mathscr{D}_{\omega}(R)$ if $\left\|\phi_{n}-\phi\right\|_{\lambda}^{(\omega)} \rightarrow 0$ as $n \rightarrow \infty$ for any $\lambda$. By the property $(\gamma)$ of $\omega, \mathscr{D}_{\omega}^{\prime}(R) \supset \mathscr{D}^{\prime}(R)$, where the elements of $\mathscr{D}^{\prime}(R)$ are the distribution in the sense of Schwarz. The family of seminorms $\left\{\phi \rightarrow\|\phi\|_{\lambda}^{(\omega)}\right\}$ on $\mathscr{D}_{\omega}(R)$ is equivalent to the family $\left\{\phi \rightarrow e^{\lambda \omega(\xi)} \sup _{\xi}|\widehat{\phi}(\xi)|\right\}$ by [19, Corollary 1.4.3].

Example 3 (see [19, Section 1.3] or [20, Section 2]). Let $P$ be the Poisson kernel for the upper half-plane.

$$
P(\xi, \eta)=\frac{\eta}{\pi} \frac{1}{\xi^{2}+\eta^{2}} .
$$

We define $u(\xi, \eta)=\int P(t-\xi, \eta) \omega(t) d t=\int P(s, \eta) \omega(s+$ $\xi) d s$ and $v(\xi, \eta)$ is the conjugate harmonic function of $u$. Let

$$
\begin{aligned}
F(\zeta)=\exp (-2 u(\xi, \eta)-2 i v(\xi, \eta)), \\
\zeta=\xi+i \eta, \eta>0 .
\end{aligned}
$$

For real $x$, define $f(x)=(2 \pi)^{-1} \int e^{i x \xi} F(\xi) d \xi$. For appropriately chosen $x_{0}, g(x)=f\left(x-x_{0}\right) f\left(-x-x_{0}\right)$ is in $\mathscr{D}_{\omega}(R)$ with support in $(-\delta, \delta)$ for any $\delta>0$.

Definition 4. $\mathscr{E}_{\omega}(R)$ is the set of all complex-valued functions $\phi$ in $R$ such that for each compact subset $K$ of $R$ the restrictions to $K$ of $\phi$ and of some $\psi \in \mathscr{D}_{\omega}(R)$ agree. The continuous linear functional of $\mathscr{E}_{\omega}(R)$ is denoted by $\mathscr{E}_{\omega}^{\prime}(R)$ whose elements are called the distribution with compact support of Beurling type.
The topology of $\mathscr{E}_{\omega}(R)$ is given by the seminorms $\phi \rightarrow$ $\inf _{\psi=\phi \text { in } K}\|\psi\|_{\lambda}^{(\omega)}$ for any $\lambda>0$ and any compact subset $K$ of $R$. We have from [19, Proposition 1.5.2] that $\mathscr{E}_{\omega}(R)$ is the set of all complex-valued functions $\phi$ in $R$ such that if $\psi \in \mathscr{D}_{\omega}(R)$, then $\psi \phi \in \mathscr{D}_{\omega}(R)$. The topology in $\mathscr{E}_{\omega}(R)$ is also given by the seminorms $\phi \rightarrow\|\psi \phi\|_{\lambda}^{(\omega)}$ for any $\lambda>0$ and any $\psi \in \mathscr{D}_{\omega}(R)$. A sequence $\left\{\phi_{n}\right\}$ is said to converge to $\phi$ in $\mathscr{E}_{\omega}(R)$ if $\| \psi \phi_{n}-$ $\psi \phi \|_{\lambda}^{(\omega)} \rightarrow 0$ as $n \rightarrow \infty$ for any $\lambda>0$ and any $\psi \in \mathscr{D}_{\omega}(R)$. $\mathscr{E}_{\omega}^{\prime}(R)$ can be identified with the set of all elements of $\mathscr{D}_{\omega}^{\prime}(R)$ which have compact support in $R$.

Definition 5. One denotes by $\mathcal{S}_{\omega}(R)$ the set of all functions $\phi \in L_{1}(R)$ with the property of $\phi$ and $\hat{\phi} \in C^{\infty}$ and for each $\lambda>0$ and $\alpha \in N$ one has

$$
\begin{aligned}
P_{\alpha, \lambda}(\phi) & =\sup _{x \in R} e^{\lambda \omega(x)}\left|\phi^{(\alpha)}(x)\right|<\infty, \\
\Pi_{\alpha, \lambda}(\phi) & =\sup _{\xi \in R} e^{\lambda \omega(\xi)}\left|\hat{\phi}^{(\alpha)}(\xi)\right|<\infty .
\end{aligned}
$$

A continuous linear functional of $\mathcal{S}_{\omega}(R)$ is denoted by $\mathcal{S}_{\omega}^{\prime}(R)$ whose elements are called the tempered distributions of Beurling type.

The topology of $\mathcal{S}_{\omega}(R)$ is defined by the seminorms $P_{\alpha, \lambda}$ and $\Pi_{\alpha, \lambda}$ for each $\lambda>0$ and $\alpha \in N$. It can be seen that $\mathscr{D}_{\omega}(R) \subset \mathcal{S}_{\omega}(R) \subset \mathscr{E}_{\omega}(R)$. A sequence $\left\{\phi_{n}\right\}$ is said to converge to $\phi$ in $\mathcal{S}_{\omega}(R)$ if $P_{\alpha, \lambda}\left(\phi_{n}-\phi\right) \rightarrow 0$ and $\Pi_{\alpha, \lambda}\left(\phi_{n}-\phi\right) \rightarrow 0$ for each $\lambda>0$ and $\alpha \in N$.

Let $T \in \mathcal{S}_{\omega}^{\prime}(R)$. We recall that $T$ is said to be periodic with period $p$ if there exists a positive real number $p$ such that $T(s)=T\left(s_{-p}\right)$ for all $s \in \mathcal{S}_{\omega}(R)$, where $s_{-p}(t)=s(t+p)$.

Definition 6. One denotes the space of periodic tempered distributions of Beurling type having a period $p$ by $\mathcal{S}_{\omega, p}^{\prime}(R)$.

Example 7. Let $\delta_{x}$ be the Dirac delta distribution such that $\delta_{x}(\phi)=\phi(x)$ and let $p>0$. Define $S_{\delta, p}=\sum_{n=-\infty}^{\infty} \delta_{n p}$. Then we have from the property $(\gamma)$ of $\omega$ that, for $\phi \in \mathcal{S}_{\omega}(R)$,

$$
\begin{aligned}
|\phi(n p)| & \leq P_{0, \lambda}(\phi) e^{-\lambda \omega(n p)} \\
& \leq P_{0, \lambda}(\phi) e^{-\lambda a}(1+|n p|)^{-\lambda b},
\end{aligned}
$$

where $a$ and $b$ are constants in the property $(\gamma)$ of $\omega$. If we take $\lambda$ such that $\lambda b>2, \sum_{n=-N}^{N} \delta_{n p}(\phi)=\sum_{n=-N}^{N} \phi(n p)$ converges in C. Since $\lim _{x \rightarrow \infty} \phi(x)=0$,

$$
\begin{aligned}
S_{\delta, p}\left(\phi_{-p}\right)= & \lim _{N \rightarrow \infty} \sum_{n=-N}^{N} \delta_{n p}\left(\phi_{-p}\right) \\
= & \lim _{N \rightarrow \infty} \sum_{n=-N}^{N} \phi((n+1) p) \\
= & \lim _{N \rightarrow \infty} \sum_{k=-N+1}^{N-1} \phi(k p)+\lim _{N \rightarrow \infty} \phi(N p) \\
& +\lim _{N \rightarrow \infty} \phi((N+1) p)=S_{\delta, p}(\phi) .
\end{aligned}
$$


Hence $S_{\delta, p} \in \mathcal{S}_{\omega, p}^{\prime}(R)$. As stated in [17, Example 2], $S_{\delta, p}=$ $\sum_{n=-\infty}^{\infty} \delta_{n p}$ is an impulse train and is useful in modelling the operation of sampling a continuous signal at sampling interval $P$ and in establishing the link between analog and digital signals via the Sampling Theorem.

We introduce the space of periodic ultradifferentiable functions with compact support.

Definition 8. For $p>0$, one denotes $\mathscr{E}_{\omega, p}$ by the set of all functions in $\mathscr{E}_{\omega}(R)$ which have period $p$. We denote $\mathscr{E}_{\omega, p}^{\prime}$ by the space of all continuous, $p$-periodic, linear mapping from $\mathscr{E}_{\omega, p}$ into $C$.

$\mathscr{E}_{\omega, p}^{\prime}$ is a vector space over $C$ when addition of functions and scalar multiplication of functions are defined in the usual manner. The topology in $\mathscr{E}_{\omega, p}$ is given by the seminorms $f \rightarrow$ $\|f \phi\|_{\lambda}^{(\omega)}$ for any $\lambda>0$ and any $\phi \in \mathscr{D}_{\omega}(R)$. A sequence $\left\{f_{n}\right\} \in$ $\mathscr{E}_{\omega, p}$ converges to $f$ in $\mathscr{E}_{\omega, p}$ if and only if $f_{n}$ and $f$ have period and $f_{n}$ converges to $f$ in $\mathscr{E}_{\omega}(R)$.

\section{Relationship between $\mathcal{S}_{\omega}(R)$ and $\mathscr{E}_{\omega, p}$ and between $\mathcal{S}_{\omega, p}^{\prime}(R)$ and $\mathscr{E}_{\omega, p}^{\prime}$}

In this section, we show that the mapping $\mathcal{S}_{\omega}(R) \rightarrow \mathscr{E}_{\omega, p}$ is linear, onto, and continuous and the mapping $\mathcal{S}_{\omega, p}^{\prime}(R) \rightarrow$ $\mathscr{E}_{\omega, p}^{\prime}$ is linear and onto. First, we show that $\mathscr{E}_{\omega, p}$ is generated by $\mathcal{S}_{\omega}(R)$.

Lemma 9. Let $p>0$ and let $\phi \in \mathcal{S}_{\omega}(R)$. For each natural number $N$, define a mapping $\phi_{N}: R \rightarrow C$ by

$$
\phi_{N}(t)=\sum_{n=-N}^{N} \phi(t-n p) .
$$

Then there exists a function $f \in \mathscr{E}_{\omega, p}$ such that, for each integer $k$, the sequences $\left\{\phi_{N}^{(k)}\right\}$ and $\left\{\widehat{\phi}_{N}^{(k)}\right\}$ converge to $f^{(k)}$ and $\widehat{f}^{(k)}$ uniformly on compact subsets of $R$, respectively.

Proof. Fix $k \in Z_{+}$. For each $t \in R$,

$$
\left|\phi^{(k)}\right| \leq e^{-k^{\prime} \omega(t)} P_{k, k^{\prime}}(\phi),
$$

where $k^{\prime}$ is taken such that $k^{\prime} b>2$ for a constant $b$ in the property $(\gamma)$ of $\omega$. Fix $l>0$ and let $N_{l}$ be positive integer such that $N_{l} p>l$. Then for $|t| \leq l$ and $n>N_{l},|n p-t| \geq n p-l>$ $p\left(n-N_{l}\right)$. For any $\epsilon>0$, let $M_{\epsilon}$ be positive integer such that $M_{\epsilon}>N_{l}$ and

$$
2 P_{k, k^{\prime}}(\phi) p^{-k^{\prime} b} e^{-k^{\prime} a} \sum_{n=M_{e}}^{\infty} \frac{1}{\left(1+\left|n-N_{l}\right|\right)^{k^{\prime} b}}<\epsilon .
$$

Here $a$ is a constant in the property $(\gamma)$ of $\omega$. Then for $|t| \leq l$ and for $L \geq K>M_{\epsilon}$, we have from the property $(\gamma)$ of $\omega$ that

$$
\begin{aligned}
& \left|\phi_{K}^{(k)}(t)-\phi_{L}^{(k)}(t)\right| \\
& \quad \leq \sum_{n=-L}^{-K}\left|\phi^{(k)}(t-n p)\right|+\sum_{n=K}^{L}\left|\phi^{(k)}(t-n p)\right| \\
& \quad \leq 2 P_{k, k^{\prime}}(\phi) \sum_{n=K}^{L} e^{-k^{\prime} \omega(t-n p)} \\
& \quad \leq 2 P_{k, k^{\prime}}(\phi) \sum_{n=M_{\epsilon}}^{\infty} e^{-k^{\prime}\left(a+b \log \left(1+p\left(n-N_{a}\right)\right)\right)} \\
& \quad \leq 2 P_{k, k^{\prime}}(\phi) p^{-k^{\prime} b} e^{-k^{\prime} a} \sum_{n=M_{\epsilon}}^{\infty} \frac{1}{\left(1+\left|n-N_{l}\right|\right)^{k^{\prime} b}}<\epsilon .
\end{aligned}
$$

Hence $\left\{\phi_{N}^{(k)}(t)\right\}$ is a Cauchy sequence, uniformly in $|t| \leq l$. Since $\delta_{\omega}(R)$ is complete, for each $k \in Z_{+}$, there exist a function $f_{k}: R \rightarrow C$ such that, for each $t \in R, \lim _{N \rightarrow \infty} \phi_{N}^{(k)}(t)$ converges to $f_{k}(t)$ uniformly on compact subsets of $R$. If we take $f=f_{0}$, we have from basic theorem for calculus and mathematical induction that $f_{0}^{(k)}=f_{k}$ for all $k \in Z_{+}$. By the same process of periodicity of $S_{\delta, p}$ in Example 7, we know that $f$ is periodic. Now since

$$
\begin{aligned}
& \left\|\psi \phi_{N}\right\|_{\lambda}^{(\omega)} \leq C \sup _{\xi} e^{\lambda \omega(\xi)}\left|\widehat{\psi \phi_{N}}(\xi)\right|=C(2 \pi)^{-1} \\
& \cdot e^{\lambda \omega(\xi)}\left|\widehat{\psi} * \widehat{\phi}_{N}(\xi)\right|=C(2 \pi)^{-1} \\
& \cdot e^{\lambda \omega(\xi)}\left|\int \widehat{\psi}(\xi-\eta) \widehat{\phi}_{N}(\eta) d \eta\right| \leq C(2 \pi)^{-1} \\
& \cdot\left|\int \widehat{\psi}(\xi-\eta) e^{\lambda \omega(\xi-\eta)} e^{\lambda \omega(\eta)} \cdot \widehat{\phi}_{N}(\eta) e^{l \omega(\eta)} e^{-l \omega(\eta)} d \eta\right| \\
& =C(2 \pi)^{-1} \Pi_{0, \lambda}(\psi) \Pi_{0, l}\left(\phi_{N}\right)\left|\int e^{(\lambda-l) \omega(\eta)} d \eta\right|,
\end{aligned}
$$

for some constant $C$, any constant $l$, and any $\psi \in \mathscr{D}_{\omega}(R)$, if we take $l$ sufficiently large, $\left\|\psi \phi_{N}\right\|_{\lambda}^{(\omega)}$ is uniformly bounded. Then since

$$
\|\psi f\|_{\lambda}^{(\omega)}=\left\|\psi\left(\lim _{N \rightarrow \infty} \phi_{N}\right)\right\|_{\lambda}^{(\omega)}=\lim _{N \rightarrow \infty}\left\|\psi \phi_{N}\right\|_{\lambda}^{(\omega)}
$$

is bounded, $f \in \mathscr{E}_{\omega}(R)$. If we replace $P_{k, k^{\prime}}$ by $\Pi_{k, k^{\prime}}$ in the proof of first part of this theorem and use

$$
\begin{aligned}
& \left\|\psi \widehat{\phi}_{N}\right\|_{\lambda}^{(\omega)}=C(2 \pi)^{-1} e^{\lambda \omega(\xi)}\left|\widehat{\psi}^{(\omega)} \widehat{\hat{\phi}}_{N}(\xi)\right| \\
& \leq C\left|\int \widehat{\psi}(\xi-\eta) e^{\lambda \omega(\xi-\eta)} e^{\lambda \omega(\eta)} \check{\phi}_{N}(\eta) e^{l \omega(\eta)} e^{-l \omega(\eta)} d \eta\right| \\
& =C \Pi_{0, \lambda}(\psi) P_{0, l}\left(\phi_{N}\right)\left|\int e^{(\lambda-l) \omega(\eta)} d \eta\right|,
\end{aligned}
$$


we can show from the same process of the above that there exist $g \in \mathscr{E}_{\omega, p}$ such that

$$
\lim _{N \rightarrow \infty} \widehat{\phi}_{N}^{(k)}(\xi)=g^{(k)}(\xi) .
$$

By the Lebesgue dominated convergence theorem, we have

$$
\begin{aligned}
g^{(k)}(\xi) & =\lim _{N \rightarrow \infty} D_{\xi}^{(k)} \int e^{-i t \xi} \phi_{N}(t) d t \\
& =D_{\xi}^{(k)} \int e^{-i t \xi} \lim _{N \rightarrow \infty} \phi_{N}(t) d t \\
& =D_{\xi}^{(k)} \int e^{-i t \xi} f(t) d t=\widehat{f}^{(k)}(\xi) .
\end{aligned}
$$

Definition 10. Define $L_{\omega}: \mathcal{\delta}_{\omega}(R) \rightarrow \mathscr{E}_{\omega, p}$ by $L_{\omega}(\phi)=f$, where $f$ is in Lemma 9, and denote $\Gamma_{\omega, p}=\left\{\gamma \in \mathcal{S}_{\omega}: L_{\omega}(\gamma)=\right.$ $1\}$.

Example 11. Choose $\epsilon>0$ such that $[-p / 4, p / 4]+[0,4 \epsilon] c$ $[-p / 2, p / 2]$. By Example 3, we can find a nontrivial $\psi \in$ $\mathscr{D}_{\omega}((0, \epsilon))$. Then $\psi * \check{\psi}$ is nontrivial and $\psi * \check{\psi} \subset \mathscr{D}_{\omega}((0,2 \epsilon))$ by [19, Corollary 1.3.14]; hence $\overline{\psi * \check{\psi}} \subset \mathscr{D}_{\omega}((0,2 \epsilon))$. If we define

$$
\varphi=|\psi * \check{\psi}|^{2}=(\psi * \check{\psi})(\overline{\psi * \check{\psi}}),
$$

we have from [19, Proposition 1.3.5] that $\varphi \subset \mathscr{D}_{\omega}((0,2 \epsilon))$. Since $\varphi$ is nonnegative and nontrivial, we have $\int \varphi(x) d x=1$ without loss of the generality by multiplying $\varphi$ by a suitable positive constant. Let

$$
m(x)=1_{[-p / 4, p / 4]+[0,2 \epsilon]} * \varphi(x),
$$

where $1_{K}$ is the characteristic function on $K$. Then $m(x)=$ 1 on $[-p / 4, p / 4]$ and $m \in \mathscr{D}_{\omega}([-p / 2, p / 2])$ by the fact that $\operatorname{supp} m \subset[-p / 4, p / 4]+[0,4 \epsilon] \subset[-p / 2, p / 2]$. Define $\widetilde{m}:$ $R \rightarrow R$ by $\widetilde{m}(x)=(m(x)+1) / 2$ and define $\chi: R \rightarrow R$ by

$$
\chi(x)= \begin{cases}\widetilde{m}(x) & x \in\left[-\frac{p}{2}, \frac{p}{2}\right] \\ \widetilde{m}(x-p)+1 & x \in\left[p, \frac{p}{2}\right] \\ \widetilde{m}(x+p)+1 & x \in\left[-p,-\frac{p}{2}\right] \\ 0 & \text { otherwise. }\end{cases}
$$

Then $\chi \in \mathscr{D}_{\omega}(R) \subset \mathcal{S}_{\omega}(R)$ and supp $\chi \subset[-p, p]$. If we define a mapping $\chi_{N}: R \rightarrow C$ by $\chi_{N}(x)=\sum_{n=-N}^{N} \chi(x-$ $n p)$, then $\lim _{N \rightarrow \infty} \chi(x)=f(x)=L_{\omega}(\chi)$ by Lemma 9. Then we have from the last part of [14, Example 18] that $L_{\omega}(\chi)=$ $f(x)=1$ for all $t \in R$.

Theorem 12. Given $f \in \mathscr{E}_{\omega, p}$, there exist $\phi \in \mathcal{S}_{\omega}(R)$ such that $f(x)=\lim _{N \rightarrow \infty} \phi_{N}(x)$ uniformly on compact subsets in $R$.
Proof. Let $\chi$ be the function in Example 11 and let $\phi=\chi f$. Since $\chi \in \mathscr{D}_{\omega}(R)$ and $f \in \mathscr{E}_{\omega}(R), \phi \in \mathscr{D}_{\omega}(R) \subset \mathcal{S}_{\omega}(R)$ and for $x$ in $R$, we have from Example 11 and Lemma 9 that

$$
\begin{aligned}
\lim _{N \rightarrow \infty} \phi_{N}(x) & =\lim _{N \rightarrow \infty} \sum_{n=-N}^{N} \chi(x-n p) f(x-n p) \\
& =f(x) \lim _{N \rightarrow \infty} \sum_{n=-N}^{N} \chi(x-n p)=f(x) .
\end{aligned}
$$

The convergence in (19) is uniform on compact subsets in $R$ by Lemma 9.

Now, we show the relationship between $\mathcal{S}_{\omega}(R)$ and $\mathscr{E}_{\omega, p}$. Since $L_{\omega}(\phi)=L_{\omega}\left(\phi_{n p}\right)$ for any $n \in Z_{+}, L_{\omega}$ is not one-toone, but $L_{\omega}$ satisfies the properties of isomorphism except for one-to-one property.

Theorem 13. The mapping $L_{\omega}: \mathcal{S}_{\omega}(R) \rightarrow \mathscr{E}_{\omega, p}$ is linear, onto, and continuous.

Proof. $L_{\omega}$ is clearly linear. Let $\chi$ be in Example 11 and let $g \in$ $\mathscr{E}_{\omega, p}$. Since $g \in \mathscr{E}_{\omega}(R)$ and $\chi \in \mathscr{D}_{\omega}(R), g \chi \in \mathscr{D}_{\omega}(R) \subset \mathcal{S}_{\omega}(R)$. Then since

$$
\begin{aligned}
L_{\omega}(g \chi)(x) & =\lim _{N \rightarrow \infty} \sum_{N=-n}^{n} g(x-n p) \chi(x-n p) \\
& =g(x) \lim _{N \rightarrow \infty} \chi_{N}(x)=g(x),
\end{aligned}
$$

$L_{\omega}$ is onto. To show that $L_{\omega}$ is continuous, let $\left\{\phi_{n}\right\}$ and $\phi$ be in $\mathcal{S}_{\omega}(R)$ such that $\left\{\phi_{n}\right\} \rightarrow \phi$ in $\mathcal{S}_{\omega}(R)$. Let $f_{n}=L_{\omega}\left(\phi_{n}\right)$ and $f=L_{\omega}(\phi)$. Since $\mathcal{S}_{\omega}(R)$ and $\mathscr{E}_{\omega, p}$ are metric spaces, we need to show that $f_{n} \rightarrow f$ in $\mathscr{E}_{\omega, p}$. But $f_{n}$ and $f$ are clearly periodic by the proof of Lemma 9 , it suffices to show that $f_{n} \rightarrow f$ in $\mathscr{E}_{\omega}(R)$. For any $\epsilon>0$, there exist $M>0$ such that if $n>M$, then $\Pi_{0,0}\left(\phi_{n}-\phi\right)<\epsilon / 6$ and can take $\Lambda$ with $\Lambda b>2$ such that

$$
\Pi_{0, \Lambda}\left(\phi_{n}-\phi\right) e^{-\Lambda a} \sum_{|m|>1} \frac{1}{(1+|m-1| p)^{\Lambda b}}<\frac{\epsilon}{2},
$$

where $a$ and $b$ are constants in the property $(\gamma)$ of $\omega$. Then for $n>M$ and $|\xi| \leq p$,

$$
\begin{aligned}
& \left|\widehat{f}_{n}(\xi)-\widehat{f}(\xi)\right| \leq \sum_{m=-\infty}^{\infty}\left|\widehat{\phi}_{n}(\xi-m p)-\widehat{\phi}(\xi-m p)\right| \\
& \leq \sum_{m=-1}^{1}\left|\widehat{\phi}_{n}(\xi-m p)-\widehat{\phi}(\xi-m p)\right| \\
& \quad+\sum_{|m|>1}\left|\widehat{\phi}_{n}(\xi-m p)-\widehat{\phi}(\xi-m p)\right| \\
& \leq 3 \Pi_{0,0}\left(\phi_{n}-\phi\right)+\sum_{|m|>1} \Pi_{0, \Lambda}\left(\phi_{n}-\phi\right) e^{-\Lambda \omega(\xi-m p)} \\
& \leq 3 \Pi_{0,0}\left(\phi_{n}-\phi\right) \\
& \quad+\Pi_{0, \Lambda}\left(\phi_{n}-\phi\right) e^{-\Lambda a} \sum_{|m|>1} \frac{1}{(1+|m-1| p)^{\Lambda b}}<\epsilon .
\end{aligned}
$$


Then $\widehat{f}_{n}(\xi)$ converges uniformly to $\widehat{f}(\xi)$ on $|\xi|<p$ and hence on $R$ by periodicity of $\widehat{f}_{n}$ and $\widehat{f}$ by Lemma 9 . Hence if we apply the Lebesgue convergence theorem, we have that, for $\varphi \in \mathscr{D}_{\omega}(R)$,

$$
\begin{aligned}
\| \varphi & \left.\left(f_{n}-f\right) \|_{\lambda}^{(\omega)}=\int \mid \varphi \widehat{\left(f_{n}-f\right.}\right)(\xi) \mid e^{\lambda \omega(\xi)} d \xi \\
& =(2 \pi)^{-1} \int\left|\left(\widehat{\varphi} *\left(\widehat{f}_{n}-\widehat{f}\right)\right)(\xi)\right| e^{\lambda \omega(\xi)} d \xi \longrightarrow 0,
\end{aligned}
$$

as $n \rightarrow \infty$. Thus $f_{n}$ converges to $f$ in $\mathscr{E}_{\omega}(R)$.

We show the relationship between periodic tempered distributions of Beurling type, $\mathcal{S}_{\omega, p}^{\prime}(R)$, and the dual space of $\mathscr{E}_{\omega, p}$

Definition 14. One denotes $\mathscr{E}_{\omega, p}^{\prime}$ by the space of all continuous, $p$-periodic linear mappings $\Lambda_{\omega}: \mathscr{E}_{\omega, p} \rightarrow C$.

In Definition 14, $p$-periodicity of $\Lambda_{\omega}$ means that $\Lambda_{\omega}(f)=$ $\Lambda_{\omega}\left(f_{-p}\right)$ for all $f \in \mathscr{E}_{\omega, p}$.

Example 15. Consider the function $\Lambda_{\omega}: \mathscr{E}_{\omega, p} \rightarrow C$ defined by $\Lambda_{\omega}(f)=f(0)$ for all $f \in \mathscr{E}_{\omega, p}$. Clearly, $\Lambda_{\omega}$ is periodic and linear. Let $f_{n} \rightarrow f$ in $\mathscr{E}_{\omega, p}$. Then $f_{n} \rightarrow f$ in $\mathscr{E}_{\omega}(R)$, hence $\varphi f_{n} \rightarrow \varphi f$ in $\mathscr{D}_{\omega}(R)$ for $\varphi \in \mathscr{D}_{\omega}(R)$. we have from [20, Proposition 3.9] that $\varphi f_{n} \rightarrow \varphi f$ in $C_{c}^{\infty}$, that is, $\varphi f_{n} \rightarrow$ $\varphi f$ uniformly on compact subset of $R$, that is, $\varphi(0) f_{n}(0) \rightarrow$ $\varphi(0) f(0)$. If we take $\varphi \in \mathscr{D}_{\omega}(R)$ with $\varphi(0) \neq 0, \Lambda_{\omega}\left(f_{n}\right)=$ $f_{n}(0) \rightarrow \Lambda_{\omega}(f)=f(0)$ in $C$. Thus $\Lambda_{\omega} \in \mathscr{E}_{\omega, p}^{\prime}$.

Let $f, g \in \mathscr{E}_{\omega}(R)$ and let $\varphi \in \mathscr{D}_{\omega}(R)$. Since $(\varphi f)^{\prime}$ and $\varphi^{\prime} f$ are in $\mathscr{D}_{\omega}(R)$ by [19, Theorem 1.3.27], $\varphi f^{\prime} \in \mathscr{D}_{\omega}(R)$ by Leibniz's formula. Hence $f^{\prime} \in \mathscr{E}_{\omega}(R)$ and $f^{(k)} \in \mathscr{E}_{\omega}(R)$ by mathematical induction and Leibniz's formula. Also clearly $f g \in \mathscr{E}_{\omega}(R)$. Then we can define the following: let $\Lambda_{\omega} \in$ $\mathscr{E}_{\omega, p}^{\prime}$ and let $a \in R, k \in Z_{+}$, and $g \in \mathscr{E}_{\omega, p}$. For all $f \in$ $\mathscr{E}_{\omega, p}, \Lambda_{\omega, a}(f)=\Lambda_{\omega}\left(f_{-a}\right), \widetilde{\Lambda}_{\omega}(f)=\Lambda_{\omega}(\widetilde{f}), \Lambda_{\omega}^{(k)}(f)=$ $(-1)^{k} \Lambda_{\omega}\left(f^{(k)}\right)$, and $\left(g \Lambda_{\omega}\right)(f)=\Lambda_{\omega}(g f)$.

Now we will show the relationship between $\mathcal{S}_{\omega, p}^{\prime}(R)$ and $\mathscr{E}_{\omega, p}^{\prime}$

Definition 16. The mapping $\Psi_{\omega}: \mathscr{E}_{\omega, p}^{\prime} \rightarrow \mathcal{S}_{\omega}^{\prime}(R)$ is defined by $\Psi_{\omega}\left(\Lambda_{\omega}\right)=\Lambda_{\omega} \circ L_{\omega}$, where $L_{\omega}$ is in Definition 14. Let $\chi$ be in Example 11. The mapping $\Phi_{\omega}: \mathscr{E}_{\omega, p}^{\prime} \rightarrow \mathcal{S}_{\omega}^{\prime}(R)$ is defined by $\Phi_{\omega}(T)(f)=T(f \chi)$ for all $f \in \mathcal{S}_{\omega}(R)$.

Clearly we have that $\Psi_{\omega}(f)$ has period $p$ and $\Psi_{\omega}$ is linear.

Theorem 17. Let $T \in \mathcal{S}_{\omega, p}^{\prime}(R)$ and let $\chi$ be the function defined in Example 11. Then the mapping $\Phi_{\omega}: \mathcal{S}_{\omega, p}^{\prime}(R) \rightarrow \mathscr{E}_{\omega, p}^{\prime}$ defined by

$$
\Phi_{\omega}(T)(f)=T(f \chi)
$$

for all $f \in \mathscr{E}_{\omega, p}$ is linear and onto.

Proof. Clearly $\Phi_{\omega}(T)$ is periodic. Now we show that $\Phi_{\omega}(T)$ is continuous. Let $\left\{f_{n}\right\}, f \in \mathscr{E}_{\omega, p}$ and let $f_{n} \rightarrow f$ in $\mathscr{E}_{\omega, p}$. Since $\left\{f_{n}\right\}, f \in \mathscr{E}_{\omega}(R)$, and $\chi \in \mathscr{D}_{\omega}(R), f_{n} \chi$ and $f \chi$ are in fact in $\mathscr{D}_{\omega}(R)$. Then by [20, Proposition 3.9] $f_{n} \rightarrow f$ in $C_{c}^{\infty}(K)$ for some compact subset $K$ of $R$; hence $\left(f_{n} \chi\right)^{(k)} \rightarrow(f \chi)^{(k)}$ uniformly on $K$ as $n \rightarrow \infty$ for any $k \in Z_{+}$. Then for any $k \in Z_{+}$and $\lambda>0$, if we let supp $\chi=K$, then

$$
\begin{aligned}
P_{\lambda, k}\left(f_{n} \chi-f \chi\right) & =\sup _{x \in R} e^{\lambda \omega(x)}\left|\left(f_{n} \chi-f \chi\right)^{(k)}(x)\right| \\
& \leq e^{\lambda \omega\left(c_{K}\right)} \sup _{x \in K}\left|\left(f_{n} \chi-f \chi\right)^{(k)}(x)\right| \\
& \longrightarrow 0,
\end{aligned}
$$

as $n \rightarrow 0$, where $c_{K}=\max _{x \in K}|x|$.

Since $f_{n} \rightarrow f$ in $\mathscr{D}_{\omega}(R)$ we have from [19, Definition 1.3.25] that, for any $\lambda>0$,

$$
\sup _{\xi \in R} e^{\lambda \omega(\xi)} \mid\left(f_{n} \chi-f \chi \widehat{)}(\xi) \mid \longrightarrow 0 \quad(n \longrightarrow \infty) .\right.
$$

Then, for any $k \in Z_{+}$and $\lambda>0$, if we let $\operatorname{supp} \chi=K$, then, by (26),

$$
\begin{aligned}
& \Pi_{\lambda, k}\left(f_{n} \chi-f \chi\right)=\sup _{\xi \in R} e^{\lambda \omega(\xi)}\left|\left(f_{n} \chi-f \chi\right)^{-(k)}(\xi)\right| \\
& =\sup _{\xi \in R} e^{\lambda \omega(\xi)}\left|\int_{K} e^{-i x \xi} x^{k}\left(f_{n} \chi-f \chi\right)(x) d x\right| \\
& \quad \leq C_{K, k} \sup _{\xi \in R} e^{\lambda \omega(\xi)}\left|\int_{K} e^{-i x \xi}\left(f_{n} \chi-f \chi\right)(x) d x\right| \\
& \quad \leq C_{K, k} \sup _{\xi \in R} e^{\lambda \omega(\xi)} \mid\left(f_{n} \chi-f \chi \widehat{)}(\xi) \mid \longrightarrow 0,\right.
\end{aligned}
$$

as $n \rightarrow \infty$, where $C_{K, k}=\sup _{x \in R}|x|^{k}$. Hence $f_{n} \chi \rightarrow f \chi$ in $\mathcal{S}_{\omega}(R)$. By the continuity of $T$,

$$
\begin{aligned}
\Phi_{\omega}(T)\left(f_{n}\right) & =T\left(f_{n} \chi\right) \longrightarrow \\
T(f \chi) & =\Phi_{\omega}(T)(f),
\end{aligned}
$$

as $n \rightarrow \infty$. Hence $\Phi_{\omega}(T)$ is continuous, that is; $\Phi_{\omega}(T)$ is in $\mathscr{E}_{\omega, p}^{\prime}$. By the same line in [17, Proof of Theorem 2], $\Phi_{\omega}(T)$ is linear and

$$
\Phi_{\omega}\left(\Psi_{\omega}\left(\Lambda_{\omega}\right)\right)=\Lambda_{\omega} ;
$$

that is, $\Phi_{\omega}(T)$ is onto.

Example 18. Let $S_{\delta, p}$ be in Example 7 and $\Lambda_{\omega}$ be in Example 15. If we choose $\chi \in \Gamma_{\omega, p}$, then for $f \in \mathscr{E}_{\omega, p}$ we have from the periodicity of $f$ that

$$
\begin{aligned}
\Phi_{\omega}\left(S_{\delta, p}\right)(f) & =\Phi_{\omega}(\chi f)=f(0) \sum_{n=-\infty}^{\infty} \chi(n p) \\
& =\Lambda_{\omega}(f) .
\end{aligned}
$$

In [17, Theorem 2], Smith showed that the mapping from periodic tempered distribution to the dual space of periodic 
infinitely differentiable functions is linear, onto, and one-toone. To guarantee one-to-one property in Theorem 17, we need to show $\phi_{N} u \rightarrow f u$ as $N \rightarrow \infty$ in $\mathcal{S}_{\omega}(R)$ for $\phi, u \in$ $\mathcal{S}_{\omega}(R)$. We can show the convergence in the seminorm $P_{\lambda, k}$ as follows.

Let $x \in R$ and take $m \in Z_{+}$such that $x \in[m p,(m+1) p]$ and let $a$ and $b$ be constants in the property $(\gamma)$ of $\omega$. Then

$$
\begin{aligned}
& \left|\phi_{N}^{(k)}(x)\right|=\left|\sum_{n=-N}^{N} \phi^{(k)}(x-n p)\right| \\
& \leq P_{\lambda, k}(\phi) \sum_{n=-\infty}^{\infty} e^{-\lambda \omega(x-n p)} \\
& \quad \leq P_{\lambda, k}(\phi)\left(\sum_{n \neq m, m+1} e^{-\lambda a} \cdot e^{-\lambda b \log (1+|x-n p|)}+2\right) \\
& \leq P_{\lambda, k}(\phi)\left(e^{-\lambda a} \frac{1}{p^{\lambda b}} \sum_{n=1}^{\infty} \frac{1}{n^{2}}+2\right)=A_{\lambda, k} .
\end{aligned}
$$

Here we take $\lambda$ such that $\lambda b>2$. By the periodicity of $f^{(k)}$ in Lemma 9, we have

$$
\left|\phi_{N}^{(k)}(x)-f^{(k)}\right| \leq A_{k}
$$

Now

$$
\begin{aligned}
& P_{\lambda, k}\left(\left(\phi_{N}-f\right) u\right)=\sup _{x \in R} e^{\lambda \omega(t)}\left|\left(\left(\phi_{N}-f\right) u\right)^{(k)}(x)\right| \\
& \leq \sup _{x \in R} e^{\lambda \omega(t)} \sum_{0 \leq j \leq k}\left|c_{k j}\left(\phi_{N}-f\right)^{(k-j)}(x) u^{(j)}(x)\right| \\
& \leq c_{k} \sup _{|x|>A} e^{\lambda \omega(t)}\left|\left(\phi_{N}-f\right)^{(k-j)}(x) u^{(j)}(x)\right| \\
& \quad+c_{k} \sup _{|x| \leq A} e^{\lambda \omega(t)}\left|\left(\phi_{N}-f\right)^{(k-j)}(x) u^{(j)}(x)\right| \\
& =I+I I,
\end{aligned}
$$

where $c_{k}=(k+1) \max _{0 \leq j \leq k}\left|c_{k j}\right|$. Using (32), for any $\epsilon>0$,

$$
\begin{aligned}
I & =c_{k} \sup _{|x|>A} e^{\lambda \omega(t)}\left|\left(\phi_{N}-f\right)^{(k-j)}(x) u^{(j)}(x)\right| \\
& \leq A_{k-j} c_{k} \sup _{|x|>A} e^{\lambda \omega(t)}\left|u^{(j)}(x)\right| \\
& \leq A_{k-j} c_{k} P_{\lambda_{u}, j}(u) e^{-\lambda_{u} \omega(x)} \\
& \leq A_{k-j} c_{k} P_{\lambda_{u}, j}(u) e^{-\lambda_{u} a} \frac{1}{(1+|x|)^{\lambda_{u} b}}<\frac{\epsilon}{2 A_{k-j} c_{k}},
\end{aligned}
$$

when we take $\lambda_{u}$ sufficiently large. Since $\phi_{N} \rightarrow f$ as $N \rightarrow \infty$ uniformly on compact subset on $R$ by Lemma 9 ,

$$
\begin{aligned}
I I & =\sup _{|x| \leq A} e^{\lambda \omega(t)}\left|\left(\phi_{N}-f\right)^{(k-j)}(x) u^{(j)}(x)\right| \\
& \leq P_{\lambda_{u}, j}(u) \sup _{|x| \leq A}\left|\left(\phi_{N}-f\right)^{(k-j)}(x)\right| \leq \frac{\epsilon}{2 c_{k}} .
\end{aligned}
$$

Hence $P_{\lambda, k}\left(\left(\phi_{N}-f\right) u\right) \rightarrow 0$ as $N \rightarrow \infty$.
But we did not succeed in the convergence of $\left(\phi_{N^{-}}-f\right) u \rightarrow$ 0 as $N \rightarrow \infty$ in the seminorm $\Pi_{\lambda, k}$, so we did not show oneto-one property in Theorem 17.

Remark 19. We have from (29) that $\Phi_{\omega} \circ \Psi_{\omega}=I$, where $I$ is the identity operator on $\mathscr{E}_{\omega, p}^{\prime}$ by onto property of $\Phi_{\omega}$, but we cannot show $\Psi_{\omega} \circ \Phi_{\omega}=I$, where $I$ is the identity operator on $\mathcal{S}_{\omega, p}^{\prime}(R)$ by the above fact about one-to-one property of $\Phi_{\omega}$.

Example 20. Let $f \in L_{1}^{\text {loc }}$ with period $p$. Then $T_{f}$ and $T_{\widehat{f}}$ are in $\mathcal{S}_{\omega, p}^{\prime}(R)$. In fact, let $\phi \in \mathcal{S}_{\omega}(R)$ and $a$ and $b$ are constants in property $(\gamma)$ of $\omega$. Then, for all $x \in R$ and any $\lambda_{1}>0$,

$$
|\phi(x)| \leq e^{-\lambda_{1} \omega(x)} P_{0, \lambda_{1}}(\phi)
$$

Hence if $n \in Z$ and $x \in[n p,(n+1) p]$,

$\phi(x)$

$$
\leq \begin{cases}e^{-\lambda_{1} \omega(n p)} P_{0, \lambda_{1}}(\phi) & n \in Z_{+} \\ e^{-\lambda_{1} \omega((n+1) p)} P_{0, \lambda_{1}}(\phi) & n \text { is negative integer. }\end{cases}
$$

Then if we take $\lambda_{1}$ sufficiently large such that $\lambda_{1} b>2$,

$$
\begin{aligned}
\left|T_{f}(\phi)\right| & \left|\int f(x) \phi(x) d x\right| \\
\leq & \sum_{n<0} \int_{n p}^{(n+1) p}|f(x)||\phi(x)| d x \\
& +\sum_{n \geq 0} \int_{n p}^{(n+1) p}|f(x)||\phi(x)| d x \\
\leq & \sum_{n<0} e^{-\lambda_{1} \omega((n+1) p)} P_{0, \lambda_{1}}(\phi) \int_{n p}^{(n+1) p}|f(x)| d x \\
& +\sum_{n \geq 0} e^{-\lambda_{1} \omega(n p)} P_{0, \lambda_{1}}(\phi) \int_{n p}^{(n+1) p}|f(x)| d x \\
\leq & \left(1+\sum_{n \leq-1} \frac{1}{(n p)^{\lambda_{1} b}}+1+\sum_{n>0} \frac{1}{(n p)^{\lambda_{1} b}}\right) \\
& \times e^{\lambda_{1} a} P_{0, \lambda_{1}}(\phi) \int_{0}^{p}|f(x)| d x \\
= & 2\left(1+\frac{1}{p^{\lambda_{1} b}} \sum_{n=1}^{\infty} \frac{1}{n^{\lambda_{1} b}}\right) e^{\lambda_{1} a} P_{0, \lambda_{1}}(\phi) \int_{0}^{p}|f(x)| d x
\end{aligned}
$$$$
<\infty \text {. }
$$

Clearly $T_{f}$ is linear and periodic by the periodicity of $f$. To show that $T_{f}$ is continuous, let $\left\{\phi_{n}\right\} \in \mathcal{S}_{\omega}(R)$ such that $\phi_{n} \rightarrow 0$ in $\mathcal{S}_{\omega}(R)$. For any $\epsilon>0$, there exists an integer $N$ 
such that $P_{0, \lambda}\left(\phi_{n}\right)<\epsilon$ for any $n>N$ and $\lambda_{1}>0$. For such an $n>N$, by (38),

$$
\begin{aligned}
& \left|\int f(x) \phi_{n}(x) d x\right| \\
& \quad \leq 2 \epsilon\left(1+\frac{1}{p^{\lambda_{1} b}} \sum_{n=1}^{\infty} \frac{1}{n^{\lambda_{1} b}}\right) e^{\lambda_{1} a} \int_{0}^{p}|f(x)| d x
\end{aligned}
$$

which shows that $T_{f}\left(\phi_{n}\right) \rightarrow 0$ as $n \rightarrow \infty$. Thus $T_{f} \in \mathcal{S}_{\omega, p}^{\prime}(R)$. By the property of Fourier transform, we have that

$$
\left|T_{\widehat{f}}(\phi)\right|=\left|\int \widehat{f}(x) \phi(x) d x\right|=\left|\int f(x) \widehat{\phi}(x) d x\right| .
$$

If we replace $P_{0, \lambda_{1}}$ by $\Pi_{0, \lambda_{2}}$ with sufficiently large $\lambda_{2}$ in (38), we have that $\left|T_{\widehat{f}}(\phi)\right|<\infty$. Through the above process, we have that $T_{\hat{f}} \in \mathcal{S}_{\omega, p}^{\prime}(R)$.

\section{Application to Fourier Analysis}

Let $\left\{T_{n}\right\}$ be a sequence in $\mathcal{S}_{\omega, p}^{\prime}(R) .\left\{T_{n}\right\}$ is said to converge provided that $\lim _{n \rightarrow \infty} T_{n}(\phi)$ exists for all $\phi \in \mathcal{S}_{\omega}(R)$. The series $\sum_{n=-\infty}^{\infty} T_{n}$ is said to converge provided that the sequence of partial sums, $\left\{\sum_{n=-N}^{N} T_{n}\right\}$, converges. Similarly, a sequence $\left\{\Lambda_{\omega, n}\right\}$ in $\mathscr{E}_{\omega, p}^{\prime}$ is said to converge provided that $\lim _{n \rightarrow \infty} \Lambda_{\omega, n}(f)$ exists for all $f \in \mathscr{E}_{\omega, p}$. The series $\sum_{n=-\infty}^{\infty} \Lambda_{\omega, n}$ is said to converge provided that the sequence of partial sums, $\left\{\sum_{n=-N}^{N} \Lambda_{\omega, n}\right\}$, converges.

Lemma 21. The mapping $\Psi_{\omega}: \mathscr{E}_{\omega, p}^{\prime} \rightarrow \mathcal{S}_{\omega, p}^{\prime}(R)$ defined by

$$
\Psi_{\omega}\left(\Lambda_{\omega}\right)=\Lambda_{\omega} \circ L_{\omega}
$$

is linear.

Proof. Since the composition of continuous linear functions, $\Psi_{\omega}\left(\Lambda_{\omega}\right) \in \mathcal{S}_{\omega}^{\prime}(R)$. Since $L_{\omega}\left(\phi_{-p}\right)=L_{\omega}(\phi)$, for any $\phi \in \mathcal{S}_{\omega}(R)$,

$$
\left(\Lambda_{\omega} \circ L_{\omega}\right)_{p}(\phi)=\Lambda_{\omega} \circ L_{\omega}\left(\phi_{-p}\right)=\Lambda_{\omega} \circ L_{\omega}(\phi) \text {. }
$$

Therefore, $\Psi_{\omega}\left(\Lambda_{\omega}\right)$ has period $P$; that is, $\Psi_{\omega}\left(\Lambda_{\omega}\right) \in$ $\mathcal{S}_{\omega, p}^{\prime}(R)$. The linearity of $\Psi_{\omega}$ is clear.

Theorem 22. $\mathcal{S}_{\omega, p}^{\prime}(R)$ and $\mathscr{E}_{\omega, p}^{\prime}$ are closed sets under convergence.

Proof. Let $\left\{T_{n}\right\}$ be a sequence in $\mathcal{S}_{\omega, p}^{\prime}(R)$ and let $T(\phi)=$ $\lim _{n \rightarrow \infty} T_{n}(\phi)$ exist for all $\phi \in \mathcal{S}_{\omega}(R)$. Since $\mathcal{S}_{\omega}(R)$ is a Frěchet space, we have from Banach-Steinhaus theorem that $T$ is linear and continuous; that is, $T \in \mathcal{S}_{\omega}^{\prime}(R)$. Since each $T_{n}$ is $P$-periodic, for each $\phi \in \mathcal{S}_{\omega}(R)$,

$$
\begin{aligned}
T_{P}(\phi) & =T\left(\phi_{-P}\right)=\lim _{n \rightarrow \infty} T_{n}\left(\phi_{-P}\right)=\lim _{n \rightarrow \infty} T_{n p}(\phi) \\
& =\lim _{n \rightarrow \infty} T_{n}(\phi)=T(\phi) .
\end{aligned}
$$

Therefore, $T \in \mathcal{S}_{\omega, p}^{\prime}(R)$. Now let $\left\{\Lambda_{\omega, n}\right\}$ in $\mathscr{E}_{\omega, p}^{\prime}$ converge and let $\Lambda_{\omega}(f)=\lim _{n \rightarrow \infty} \Lambda_{\omega, n}(f)$ for all $f \in \mathscr{E}_{\omega, p}$. For each positive integer $n$, let $T_{n}=\Psi_{\omega}\left(\Lambda_{\omega, n}\right)$. Then for any $\phi \in \mathcal{S}_{\omega}(R)$, from Lemma 21,

$$
\begin{aligned}
\lim _{n \rightarrow \infty} T_{n}(\phi) & =\lim _{n \rightarrow \infty} \Psi_{\omega}\left(\Lambda_{\omega, n}\right)(\phi) \\
& =\lim _{n \rightarrow \infty} \Lambda_{\omega, n}\left(L_{\omega}(\phi)\right)=\Lambda_{\omega}\left(L_{\omega}(\phi)\right) .
\end{aligned}
$$

Therefore, $\lim _{n \rightarrow \infty} T_{n}=\Lambda_{\omega} \circ L_{\omega}$ and $\Lambda_{\omega} \circ L_{\omega} \in \mathcal{S}_{\omega, p}^{\prime}(R)$ by (43). We have from the mathematical equation of $L_{\omega}$ in the proof of Theorem 13 that, for each $f \in \mathscr{E}_{\omega, p}$,

$$
\Phi_{\omega}\left(\Lambda_{\omega} \circ L_{\omega}\right)(f)=\Lambda_{\omega} \circ L_{\omega}(\chi f)=\Lambda_{\omega}(f) .
$$

Hence $\Phi_{\omega}\left(\Lambda_{\omega} \circ L_{\omega}\right)=\Lambda_{\omega}$; that is, $\Lambda_{\omega} \in \mathscr{E}_{\omega, p}^{\prime}$.

For any $f \in \mathscr{E}_{\omega, p}$, we have

$$
\Phi_{\omega}(T)(f)=T(f \chi)=\lim _{n \rightarrow \infty} T_{n}(f \chi)=\lim _{n \rightarrow \infty} \Phi_{\omega}\left(T_{n}\right) .
$$

Therefore,

(a) If $\left\{T_{n}\right\} \in \mathcal{S}_{\omega, p}^{\prime}(R)$ and $T=\lim _{n \rightarrow \infty} T_{n}$, then $\Phi(T)=$ $\lim _{n \rightarrow \infty} \Phi\left(T_{n}\right)$.

(b) From Theorem 22, if $\left\{\Lambda_{\omega}\right\} \in \mathscr{E}_{\omega, p}^{\prime}$ and $\Lambda_{\omega}=$ $\lim _{n \rightarrow \infty} \Lambda_{\omega, n}$, then $\Psi_{\omega}\left(\Lambda_{\omega}\right)=\lim _{n \rightarrow \infty}\left(\Lambda_{\omega, n}\right)$.

From (a) and (b), we have that $\Phi_{\omega}$ and $\Psi_{\omega}$ preserve convergence.

Example 23 (Fourier series of the (distributional) Fourier transform of an impulse train). We define the Fourier transform of $T \in \mathcal{S}_{\omega}^{\prime}(R)$ by

$$
\mathscr{F}(T)(f)=T(\mathscr{F}(f)), \quad f \in \mathcal{S}_{\omega}(R) .
$$

We have from an application of the Fubini theorem that (47) generalizes the classical Fourier transform.

For each integer $n$, define $g_{n}: R \rightarrow C$ by $g_{n}(\xi)=e^{-i n P \xi}$ for all $\xi \in R$. Then, for $\phi \in \mathcal{S}_{\omega}^{\prime}(R)$,

$$
\begin{aligned}
\mathscr{F}\left(S_{\delta, P}\right)(\phi) & =S_{\delta, P}(\mathscr{F}(\phi))=\sum_{-\infty}^{\infty} \delta_{n P}(\mathscr{F}(\phi)) \\
& =\sum_{-\infty}^{\infty} \mathscr{F}\left(\delta_{n P}\right)(\phi)=\sum_{-\infty}^{\infty} T_{g_{n}}(\phi) .
\end{aligned}
$$

Therefore, the Fourier transform of an impulse train is $\mathscr{F}\left(S_{\delta, P}\right)=\sum_{-\infty}^{\infty} T_{g_{n}}$.

\section{Conflicts of Interest}

The author declares that there are no conflicts of interest regarding the publication of this paper.

\section{Acknowledgments}

This work was supported by a grant from Inje University for the Research, 20170080. 


\section{References}

[1] A. H. Zemanian, Distribution Theory and Transform Analysis, Ma Graw-Hill Book, 1987.

[2] N. C. Dias and J. N. Prata, "A multiplicative product of distributions and a class of ordinary differential equations with distributional coefficients," Journal of Mathematical Analysis and Applications, vol. 359, no. 1, pp. 216-228, 2009.

[3] D. Judge, "On Zemanian's distributional eigenfunction transforms," Journal of Mathematical Analysis and Applications, vol. 34, pp. 187-201, 1971.

[4] S. Pilipović, "Generalization of Zemanian spaces of generalized functions which have orthonormal series expansions," SIAM Journal on Mathematical Analysis, vol. 17, no. 2, pp. 477-484, 1986.

[5] A. H. Zemanian, Generalized Integral Transformations, Interscience, New York, NY, USA, 1968.

[6] M. Craddock, "Fundamental solutions, transition densities and the integration of Lie symmetries," Journal of Differential Equations, vol. 246, no. 6, pp. 2538-2560, 2009.

[7] A. H. Zemanian, "A solution of a division problem arising from Bessel-type differential equations," SIAM Journal on Applied Mathematics, vol. 15, pp. 1106-1111, 1967.

[8] A. Napolitano, "Sampling theorems for Doppler-stretched wide-band signals," Signal Processing, vol. 90, no. 7, pp. 22762287, 2010.

[9] A. V. Oppenheim and R. W. Schafer, Discrete-Time Signal Processing, Prentice-Hall, New Jergey, NJ, USA, 1999.

[10] V. I. Gorbacuk, "On Fourier series of periodic ultradistributions," Ukrainian Mathematical Journal, vol. 34, no. 2, pp. 144150, 1982.

[11] S. Pilipović, "Structural theorems for periodic ultradistributions," Proceedings of the American Mathematical Society, vol. 98, no. 2, pp. 261-266, 1986.

[12] Y. Taguchi, "Fourier coefficients of periodic functions of Gevrey classes and ultradistributions," Yokohama Mathematical Journal, vol. 35, no. 1-2, pp. 51-60, 1987.

[13] I. Cioranescu, "The characterization of the almost periodic ultradistributions of Beurling type," Proceedings of the American Mathematical Society, vol. 116, no. 1, pp. 127-134, 1992.

[14] M. C. Gómez-Collado, "Almost periodic ultradistributions of Beurling and of Roumieu type," Proceedings of the American Mathematical Society, vol. 129, no. 8, pp. 2319-2329, 2001.

[15] A. I. Zayed, "Wavelet transform of periodic generalized functions," Journal of Mathematical Analysis and Applications, vol. 183, no. 2, pp. 391-412, 1994.

[16] D. C. Smith, "An introduction to distribution theory for signals analysis. Part II. The convolution," Digital Signal Processing, vol. 16, no. 4, pp. 419-444, 2006.

[17] D. C. Smith, "An introduction to distribution theory for signals analysis. Part III. PERiodic tempered distributions," Digital Signal Processing, vol. 41, pp. 1-21, 2015.

[18] A. Beuring, Quasi-Analyticity and General Distributions, lectures 4 and 5, A.M.S. Summmer Institute, Stanford, 1961.

[19] G. Björck, "Linear partial differential operators and generalized distributions," Arkiv för Matematik, vol. 6, pp. 351-407, 1965.

[20] R. W. Braun, R. Meise, and B. A. Taylor, "Ultradifferentiable functions and Fourier analysis," Results in Mathematics, vol. 17, no. 3-4, pp. 206-237, 1990.

[21] R. Meise and B. A. Taylor, "Whitney's extension theorem for ultradifferentiable functions of Beurling type," Arkiv för Matematik, vol. 26, no. 2, pp. 265-287, 1988. 


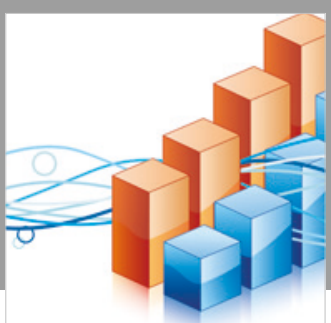

Advances in

Operations Research

\section{-n-m}
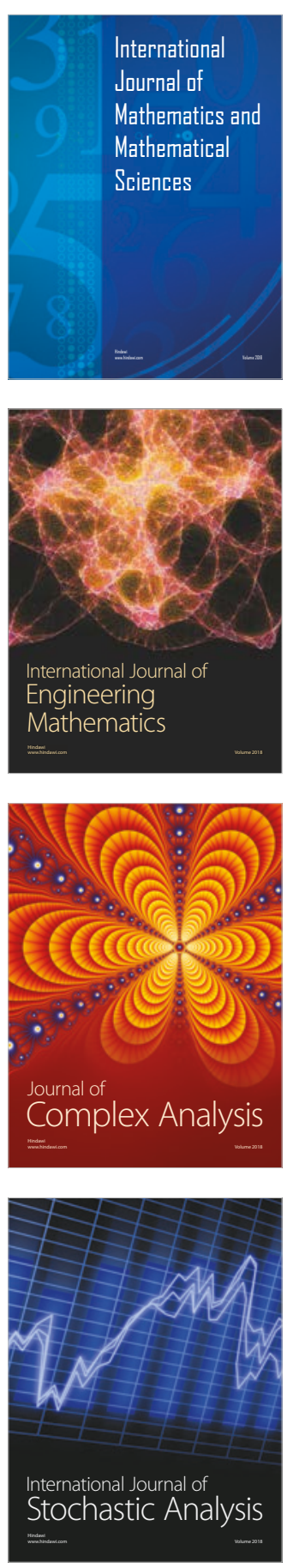
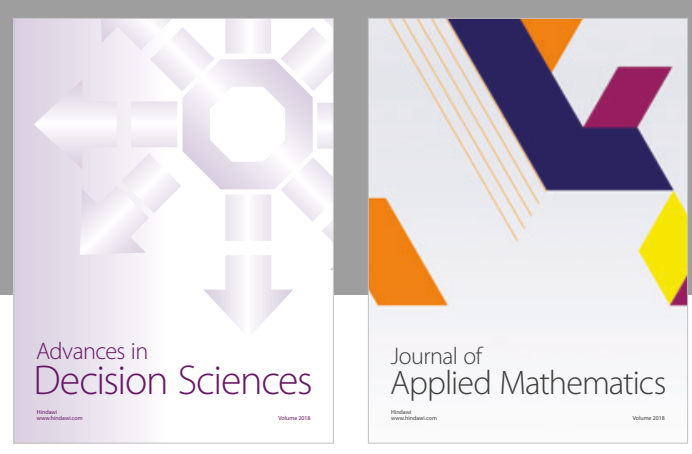

Journal of

Applied Mathematics
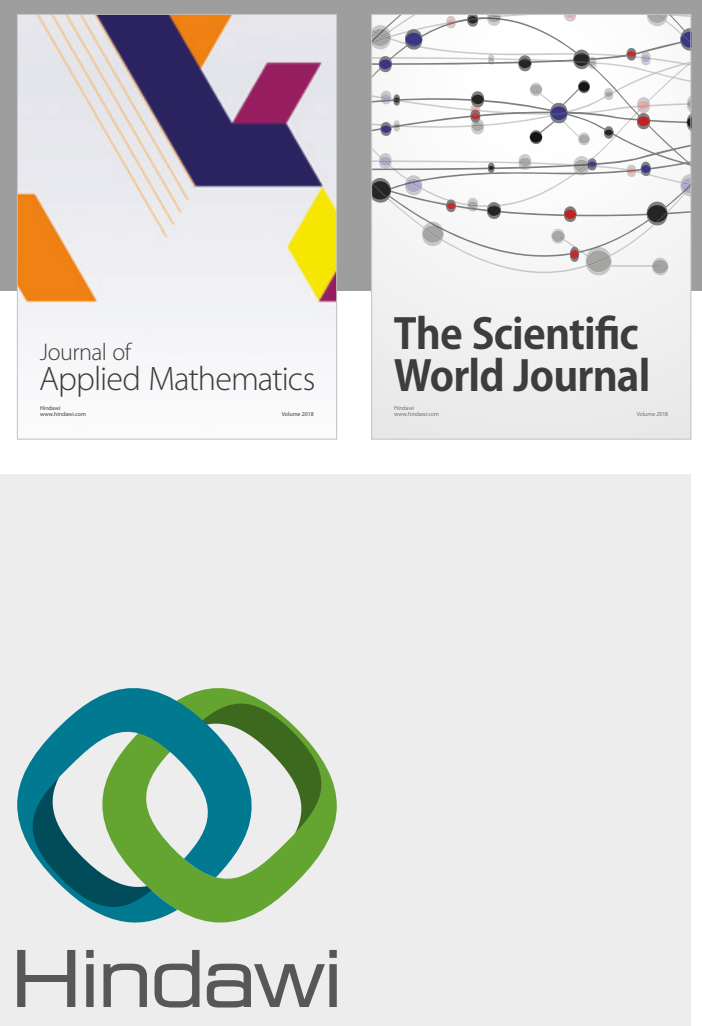

Submit your manuscripts at

www.hindawi.com

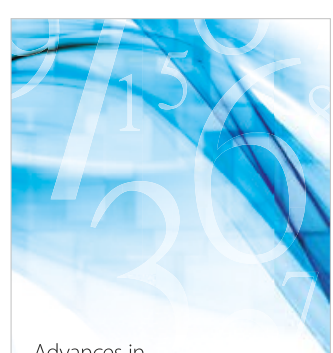

Advances in
Numerical Analysis
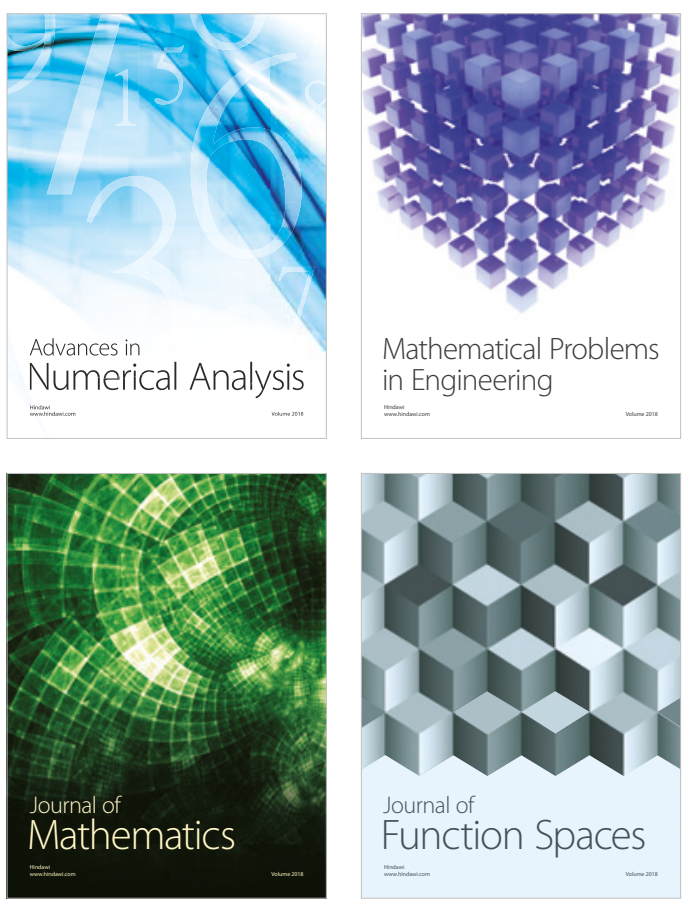

Mathematical Problems in Engineering

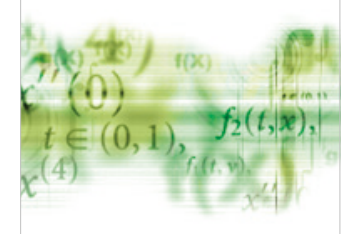

International Journal of

Differential Equations

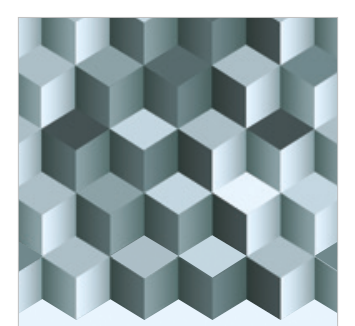

Journal of

Function Spaces

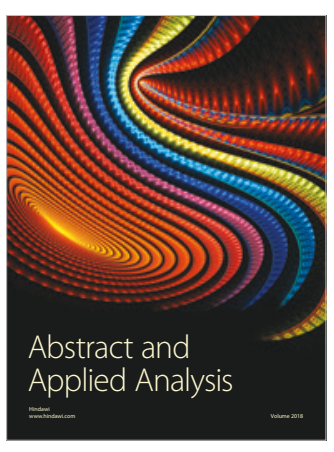

The Scientific

World Journal

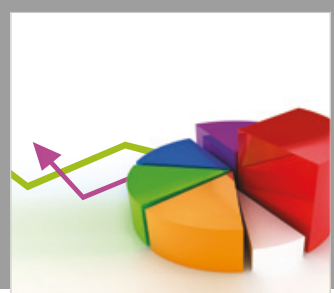

Journal of

Probability and Statistics
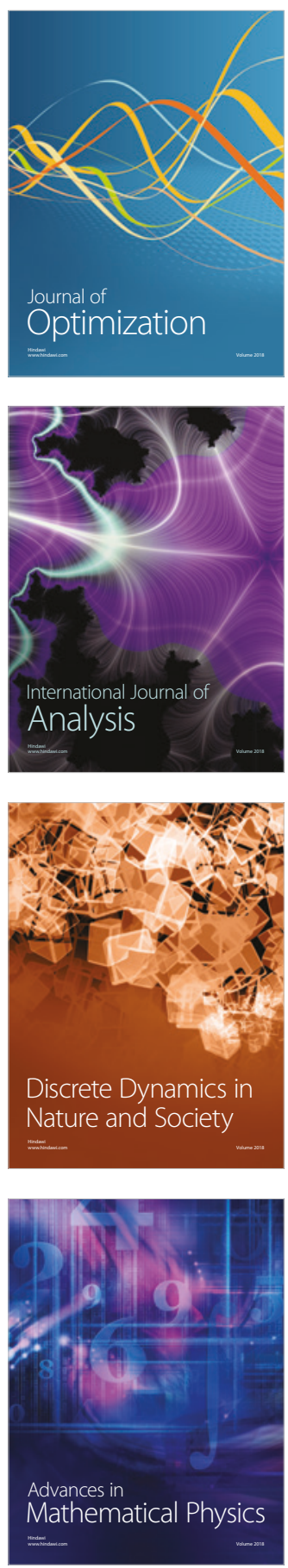